\title{
El sistema de predicción de la calidad del aire en AEMET: verificación
}

\author{
Coralina Hernández Trujillo ${ }^{1}$ (chernandezt@aemet.es) \\ Alberto Cansado Auría ${ }^{1}$ (acansadoa@aemet.es) \\ María Allué Camachoํ(malluec@aemet.es) \\ Isabel Martínez Marco ${ }^{1}$ (imartinezm@aemet.es)
}

${ }^{1}$ AEMET / Departamento de Desarrollo y Aplicaciones / Área de Aplicaciones

\begin{abstract}
RESUMEN
La verificación frente a las observaciones es una de las tareas fundamentales para poder evaluar la calidad de los pronósticos del sistema de predicción de calidad del aire de AEMET y, también, para analizar si los cambios realizados en el sistema, como por ejemplo el uso de nuevos inventarios de emisiones o la implementación de nuevas versiones del modelo, suponen una mejora en el sistema.
\end{abstract}

En este trabajo evaluaremos los resultados de la verificación del índice diario de calidad del aire previsto por AEMET, que se calcula con las salidas del modelo de transporte químico MOCAGE (Modèle de Chimie Atmosphérique de Grande Échelle), y del índice previsto a partir del postproceso para la corrección del sesgo usando el método Hybrid Forecast aplicado a la versión operativa del modelo.

PALABRAS CLAVE: calidad del aire; modelización; verificación.

\section{INTRODUCCIÓN}

La Agencia Estatal de Meteorología proporciona información sobre la composición química de la atmósfera mediante un sistema de predicción de la calidad del aire con el que se realizan predicciones operativas de diferentes contaminantes sobre la península ibérica y Baleares. Dicho sistema de predicción se basa en el modelo de transporte químico MOCAGE (Modèle de Chimie Atmosphérique de Grande Échelle), desarrollado por Météo-France y utilizado en AEMET en virtud de un acuerdo de colaboración con el citado servicio meteorológico. Actualmente, el sistema utiliza el inventario de emisiones TNO-MACCIII (2011), que es la referencia más actual en inventarios de alta resolución sobre Europa que hay disponible.

El modelo MOCAGE proporciona valores previstos de concentración de diferentes especies químicas como son el ozono $\left(\mathrm{O}_{3}\right)$, el dióxido de nitrógeno $\left(\mathrm{NO}_{2}\right)$, el monóxido de nitrógeno ( $\left.\mathrm{NO}\right)$, el monóxido de carbono $(\mathrm{CO})$ o el dióxido de azufre $\left(\mathrm{SO}_{2}\right)$ y dos variables de material particulado de tamaños menores de 10 micras y menores de 2,5 micras, el PM10 y el PM2.5 respectivamente. Además, con dichos valores se calcula un índice de calidad del aire con el objetivo de facilitar al público su comprensión e interpretación.

Sin embargo, estas predicciones pierden precisión conforme descienden a nivel local debido, principalmente, a la elevada incertidumbre que hay en los inventarios de emisiones y al incompleto conocimiento de los procesos físico-químicos que tienen lugar en la atmósfera. 
Para mejorar las predicciones de los modelos de calidad del aire, uno de los métodos más avanzados que se puede utilizar es la asimilación de datos, pero en este caso la disponibilidad limitada de observaciones en tiempo real y la complejidad que se añadiría al sistema hacen más adecuada la utilización de otras técnicas más simples que corrijan la salida directa del modelo. Entre los métodos de postproceso más sencillos que se pueden implementar se encuentra la técnica llamada Hybrid Forecast (KANG et al., 2008), en la que se utilizan las observaciones más recientes que se tengan disponibles para corregir la predicción del modelo.

En este trabajo haremos una comparación de las predicciones de la versión operativa del modelo y de una versión posprocesada mediante la técnica Hybrid Forecat, a través de la verificación (frente a los datos de observación) del índice de calidad del aire previsto por cada versión del modelo. Para ello, contamos con las observaciones de las redes de calidad del aire gestionadas por las comunidades autónomas y entidades locales, que obtenemos a través del MITECO (Ministerio para la Transición Ecológica).

\section{POSTPROCESO}

El postproceso implementado a la versión operativa del modelo (al que denominaremos híbrido o versión híbrida) está basado en la técnica llamada Hybrid Forecast. Esta metodología consiste en modificar la predicción del modelo, en los puntos de observación, a partir del valor observado en un tiempo previo, que generalmente es el dato observado 24 horas antes de la hora de la predicción. De modo que la predicción en el punto de observación se corrige sumando la diferencia entre la observación y la predicción del día anterior en dicho punto. Esto es,

$$
H F_{t+\Delta t}=M_{t+\Delta t}+\left(O_{t}-M_{t}\right)
$$

donde, $H F_{t+\Delta t}$ denota la predicción híbrida en un tiempo $t+\Delta t, O_{t}$ es la observación en tiempo $t$ y $M_{t}$ y $M_{t+\Delta t}$ son las predicciones del modelo en tiempo $t$ y $t+\Delta t$, respectivamente. En nuestro caso, hemos utilizado $\Delta t=24 \mathrm{~h}$.

\section{VERIFICACIÓN DEL ÍNDICE EUROPEO DE CALIDAD DEL AIRE}

El índice de calidad del aire previsto por AEMET se elabora diariamente a partir de los valores horarios que pronostica el modelo MOCAGE de los siguientes contaminantes: ozono $\left(\mathrm{O}_{3}\right)$, dióxido de nitrógeno $\left(\mathrm{NO}_{2}\right)$, dióxido de azufre ( $\mathrm{SO}_{2}$ ) y material particulado de tamaños menores de 10 micras (PM10) y menores de 2,5 micras (PM2.5). Acada valor de concentración de estos contaminantes se le asocia otro valor adimensional que se corresponde con una etiqueta descriptiva y un color. El índice diario previsto por AEMET se compone de cinco categorías (Muy Buena, Buena, Moderada, Mala y Muy Mala) (figura 1).

Para generar este índice, que integra la información de todos los contaminantes, es necesario calcular un índice parcial para cada una de las especies mencionadas anteriormente. De modo que, el índice final que se presenta es el resultado de seleccionar el valor máximo de los índices parciales en cada punto de rejilla.

\begin{tabular}{|ccccccc}
\hline & $\begin{array}{c}\text { Índice Europeo } \\
\text { medias }\left(\mu \mathrm{g} / \mathrm{m}^{3}\right)\end{array}$ & $\begin{array}{c}\mathbf{O}_{3} \\
\text { móvil } 8 \mathrm{~h}\end{array}$ & $\begin{array}{c}\mathbf{N O}_{2} \\
\mathrm{lh}\end{array}$ & $\begin{array}{c}\mathbf{S O}_{2} \\
\mathrm{lh}\end{array}$ & $\begin{array}{c}\text { PM10 } \\
\text { móvil 24h }\end{array}$ & $\begin{array}{c}\text { PM2.5 } \\
\text { móvil 24h }\end{array}$ \\
\hline Muy Buena & 1 & $0-80$ & $0-40$ & $0-100$ & $0-20$ & $0-10$ \\
\hline Buena & 2 & $80-120$ & $40-100$ & $100-200$ & $20-35$ & $10-20$ \\
\hline Moderada & 3 & $120-180$ & $100-200$ & $200-350$ & $35-50$ & $20-25$ \\
\hline Mala & 4 & $180-240$ & $200-400$ & $350-500$ & $50-100$ & $25-50$ \\
\hline Muy Mala & 5 & $>240$ & $>400$ & $>500$ & $>100$ & $>50$ \\
\hline
\end{tabular}

Figura 1. Criterios para el cálculo del índice diario previsto por AEMET. 
Los resultados que presentamos a continuación nos permiten analizar cómo se ajusta tanto el índice previsto por la versión operativa del modelo como el previsto por la versión híbrida a la realidad observada, para cada uno de los contaminantes que hemos mencionado. De esta manera, podremos evaluar el comportamiento de las predicciones operativas y si supone una mejora de las predicciones en los puntos de observación el postproceso aplicado al modelo.

Se ha realizado una verificación objetiva comparando el índice que se obtiene a partir de las predicciones en cada una de las estaciones de observación con el índice calculado a partir de los datos de observación de cada

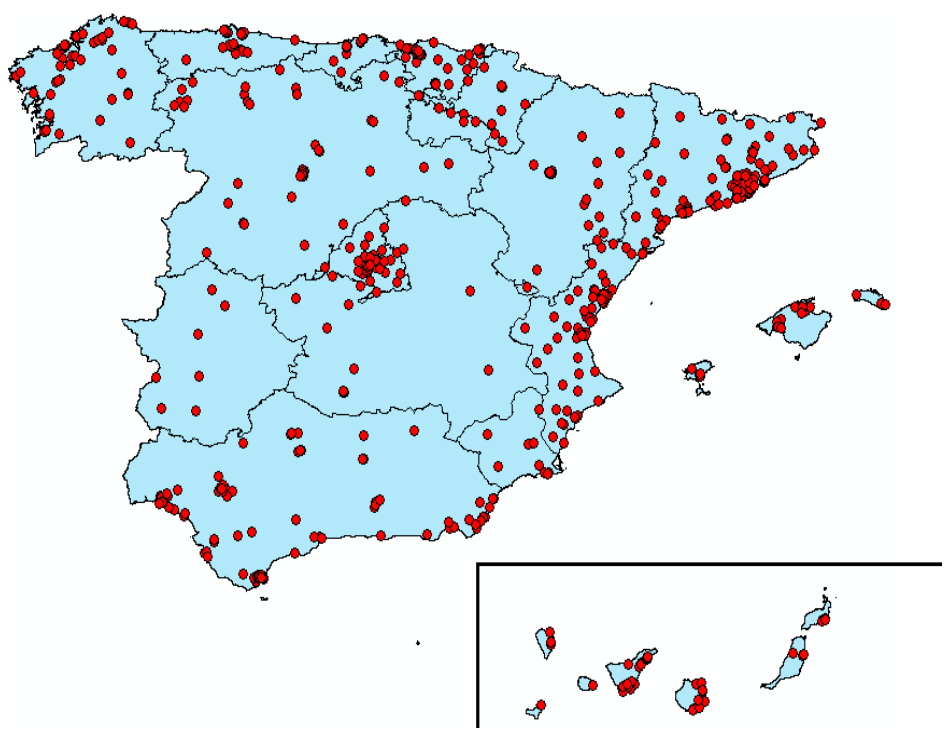

Figura 2. Estaciones de medida de la calidad del aire en España. estación (figura 2).

Los resultados de la verificación que se presenta están basados en el cálculo de medidas de exactitud y skill scores para variables multicategóricas que han surgido como generalización a los ya conocidos para variables categóricas (JolifFe y StePhEnSon, 2012). Todas las medidas calculadas se obtienen a través de tablas de contingencia que resumen el número de predicciones y observaciones en las diferentes categorías (Muy Buena, Buena, Moderada, Mala y Muy Mala). Entre las medidas que hemos decidido usar para la verificación del índice se encuentran: la proporción de predicciones correctas (PC) y la probabilidad de detección de cada categoría $\left(P O D_{i}\right)$, donde,

$$
\begin{gathered}
P C=\frac{1}{N} \cdot \sum_{i=1}^{5} n\left(F_{i}, O_{i}\right) \\
P O D_{i}=\frac{n\left(F_{i}, O_{i}\right)}{N\left(O_{i}\right)}, \quad i=1, \ldots, 5 .
\end{gathered}
$$

siendo $n\left(F_{i}, O_{i}\right)$ el número de predicciones correctas en la categoría $i$ y $N\left(O_{i}\right)$ el número de observaciones en la categoría $i$.

A continuación presentamos las tablas de contingencia que se han obtenido para cada especie $\left(\mathrm{NO}_{2}, \mathrm{SO}_{2}\right.$, $\mathrm{O}_{3}$, PM10 y PM2.5) usando la versión operativa del modelo y también considerando las predicciones calculadas con el postproceso utilizando la técnica Hybrid Forecast descrita en el apartado anterior. El periodo escogido para la realización de esta verificación es el comprendido entre el 25/08/2017 y el 25/08/2018, salvo para el material particulado (PM10 y PM2.5), que al incluirse en la versión operativa en noviembre de 2017, el periodo de verificación comienza en dicho mes.

\subsection{Resultados de la verificación para el $\mathrm{NO}_{2}$}

En los datos correspondientes la versión operativa del modelo (tabla 1 y figura 3.a) podemos observar que prácticamente el 89,79\% de ellos se encuentran en las categorías Muy Buena y Buena, donde el 61,97\% se corresponde con predicciones correctas. En las categorías Moderada, Mala y Muy Mala se cometen muy pocos errores en todo el periodo, siendo el más alto un 3,57 \% de predicciones en la categoría Moderada correspondientes a observaciones en la categoría Buena. 
Con respecto a los datos de la versión híbrida del modelo (tabla 2 y figura 3.b), destaca el aumento de predicciones correctas en la categoría Buena, pasando de un 14,96\% de aciertos en esa categoría con la versión operativa del modelo a un 28,02 \% con la versión híbrida y el descenso de los errores al predecir Muy Buena cuando se observa Buena (de un 21,18\% a un 7,77\%). También aumentan las predicciones correctas en la categoría Moderada, de un 1,47 \% a un 2,64\%, mejorando considerablemente los resultados, puesto que el porcentaje total de observaciones en dicha categoría está en torno al $5 \%$ del total de datos.

\begin{tabular}{|c|c|c|c|c|c|c|c|}
\hline & \multicolumn{5}{|c|}{ Observaciones } & \multirow[b]{2}{*}{ Total } \\
\hline & & Muy Buena & Buena & Moderada & Mala & Muy Mala & \\
\hline \multirow{5}{*}{ 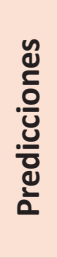 } & Muy Buena & 47,01 & 21,18 & 1,16 & 0,02 & 0,01 & 69,38 \\
\hline & Buena & 6,64 & 14,96 & 1,85 & 0,01 & 0 & 23,46 \\
\hline & Moderada & 0,8 & 3,57 & 1,47 & 0,04 & 0 & 5,88 \\
\hline & Mala & 0,03 & 0,37 & 0,64 & 0,06 & 0 & 1,1 \\
\hline & Muy Mala & 0 & 0,01 & 0,13 & 0,04 & 0 & 0,18 \\
\hline & Total & 54,48 & 40,09 & 5,25 & 0,17 & 0,01 & 100 \\
\hline
\end{tabular}

Tabla 1. Tabla de contingencia en porcentajes para el $\mathrm{NO}_{2}$ correspondiente a la versión operativa del modelo.

Por otro lado, atendiendo a la categoría Buena, podemos observar una disminución de los aciertos al usar las predicciones del modelo híbrido (pasando de un 47,01 \% con la versión operativa a un 41,48\% con la versión híbrida) y un aumento de los errores al predecir la categoría Buena cuando se observa Muy Buena (de un 6,64 \% con la versión operativa a un 11,37 \% con la versión híbrida). Este aumento de los errores en esta categoría puede deberse a que, en determinadas ocasiones, si la diferencia entre la observación y la predicción del modelo operativo del día anterior es muy grande, la predicción de la versión híbrida es bastante mayor que la predicción del modelo, lo que en algunos casos hace que la predicción de la versión híbrida aumente una o varias categorías con respecto a la predicción de la versión operativa del modelo.

\begin{tabular}{|c|c|c|c|c|c|c|c|}
\hline & \multicolumn{5}{|c|}{ Observaciones } & \multirow[b]{2}{*}{ Total } \\
\hline & & Muy Buena & Buena & Moderada & Mala & Muy Mala & \\
\hline \multirow{5}{*}{ 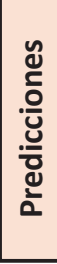 } & Muy Buena & 41,48 & 7,77 & 0,13 & 0,01 & 0 & 49,39 \\
\hline & Buena & 11,37 & 28,02 & 1,92 & 0,02 & 0 & 41,33 \\
\hline & Moderada & 0,49 & 4,98 & 2,64 & 0,05 & 0 & 8,16 \\
\hline & Mala & 0,02 & 0,27 & 0,58 & 0,09 & 0 & 0,96 \\
\hline & Muy Mala & 0 & 0,01 & 0,12 & 0,03 & 0 & 0,16 \\
\hline & Total & 53,36 & 41,05 & 5,39 & 0,2 & 0 & 100 \\
\hline
\end{tabular}

Tabla 2. Tabla de contingencia en porcentajes para el $\mathrm{NO}_{2}$ correspondiente a la versión híbrida del modelo.

Si analizamos la proporción de predicciones correctas o la probabilidad de detección de cada categoría (figuras 8 y 9), podemos ver que la versión híbrida del modelo tiene mejores resultados que la versión operativa a la hora de predecir el índice diario de calidad del aire. La proporción de predicciones correctas aumenta en la versión híbrida (de 0,63 a 0,72) y las probabilidades de detección de todas las categorías son mayores que las de la versión del modelo operativo, salvo la de la categoría Muy Buena, que es ligeramente menor con la versión híbrida (figura 9). Esto ocurre porque, como hemos comentado anteriormente, el porcentaje de predicciones correctas en esa categoría es más bajo con la versión híbrida. 
a)

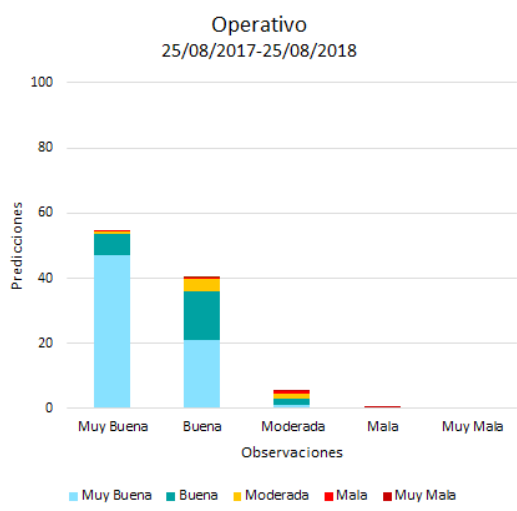

b)

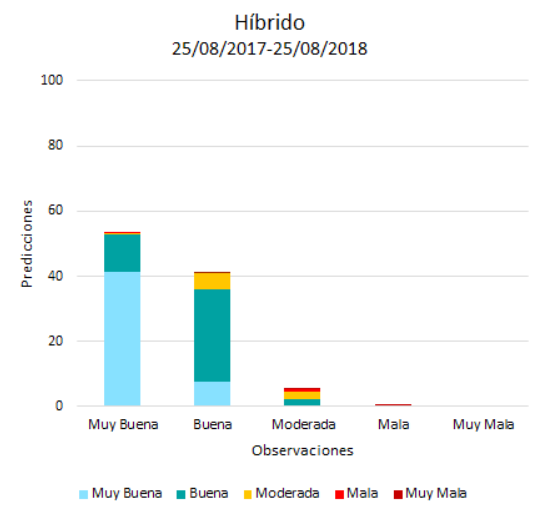

Figura 3.

Gráfico de barras correspondiente a los datos de la verificación del $\mathrm{NO}_{2}$ : a) versión operativa del modelo y b) versión híbrida del modelo.

\subsection{Resultados de la verificación para el $\mathrm{SO}_{2}$}

Los resultados de la verificación del índice para el $\mathrm{SO}_{2}$ (tablas 3 y 4 y figura 4) son bastante similares para las dos versiones del modelo que estamos comparando. En general, se obtiene un porcentaje muy alto de aciertos en la primera categoría del índice (97,25 \% y 98,44 \% con las versión operativa e híbrida, respectivamente) y muy pocos errores, de los que los más destacables son los cometidos al predecir la categoría Buena cuando se observa Muy Buena $(1,98 \%$ y $0,94 \%)$. Esto conlleva a que la proporción de predicciones correctas en ambas versiones del modelo es cercana a 1, como podemos ver en la figura $8 \mathrm{y}$, además, que las probabilidades de detección de la primera categoría sean muy altas en ambas versiones del modelo, mientras que la del resto de categorías es muy baja, al haber muy pocas observaciones en esos casos (figura 9).

El hecho de que en los últimos años se hayan reducido considerablemente los niveles de $\mathrm{SO}_{2}$ en España y que, además, en el índice europeo de calidad del aire (figura 1) concentraciones de hasta $100 \mu \mathrm{g} / \mathrm{m}^{3} \mathrm{de} \mathrm{SO}{ }_{2}$ entren dentro de la categoría Muy Buena, explica el comportamiento de los datos de la verificación del índice previsto para este contaminante.

\begin{tabular}{|c|c|c|c|c|c|c|c|}
\hline & \multicolumn{5}{|c|}{ Observaciones } & \multirow[b]{2}{*}{ Total } \\
\hline & & Muy Buena & Buena & Moderada & Mala & Muy Mala & \\
\hline \multirow{5}{*}{ 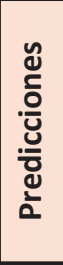 } & Muy Buena & 97,25 & 0,38 & 0,01 & 0,01 & 0,1 & 97,71 \\
\hline & Buena & 1,98 & 0,02 & 0 & 0 & 0 & 2 \\
\hline & Moderada & 0,26 & 0,01 & 0 & 0 & 0 & 0,27 \\
\hline & Mala & 0,02 & 0 & 0 & 0 & 0 & 0,02 \\
\hline & Muy Mala & 0 & 0 & 0 & 0 & 0 & 0 \\
\hline & Total & 99,51 & 0,41 & 0,06 & 0,01 & 0,01 & 100 \\
\hline
\end{tabular}

Tabla 3. Tabla de contingencia en porcentajes para el $\mathrm{SO}_{2}$ correspondiente a la versión operativa del modelo.

a)

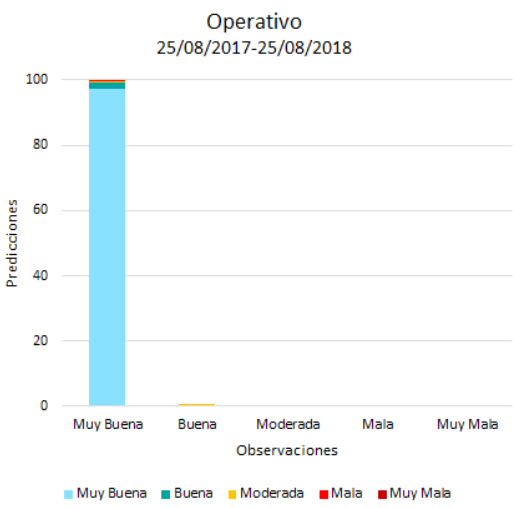

b)

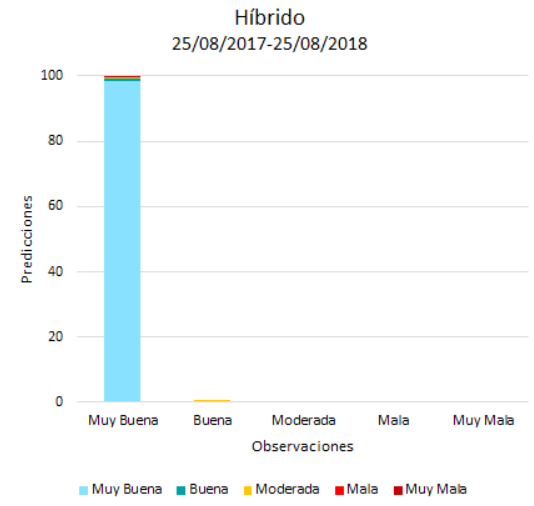

Figura 4.

Gráfico de barras correspondiente a los datos de la verificación del $\mathrm{SO}_{2}$ :

a) versión operativa del modelo y b) versión híbrida del modelo. 


\begin{tabular}{|c|c|c|c|c|c|c|c|}
\hline & \multicolumn{5}{|c|}{ Observaciones } & \multirow[b]{2}{*}{ Total } \\
\hline & & Muy Buena & Buena & Moderada & Mala & Muy Mala & \\
\hline \multirow{5}{*}{ 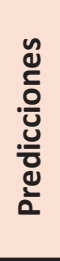 } & Muy Buena & 98,44 & 0,35 & 0,05 & 0,01 & 0,01 & 98,86 \\
\hline & Buena & 0,94 & 0,06 & 0,01 & 0 & 0 & 1,01 \\
\hline & Moderada & 0,1 & 0,01 & 0 & 0 & 0 & 0,11 \\
\hline & Mala & 0,01 & 0 & 0 & 0 & 0 & 0,01 \\
\hline & Muy Mala & 0,01 & 0 & 0 & 0 & 0 & 0,01 \\
\hline & Total & 99,5 & 0,42 & 0,06 & 0,01 & 0,01 & 100 \\
\hline
\end{tabular}

Tabla 4. Tabla de contingencia en porcentajes para el $\mathrm{SO}_{2}$ correspondiente a la versión híbrida del modelo.

\subsection{Resultados de la verificación para el PM10}

En las tablas de contingencia para el PM10 (tablas 5 y 6) vemos diferencias muy claras entre ambas versiones del modelo. En general, el porcentaje total de predicciones de la versión operativa en la categoría Muy Buena es muy alto (92,59\%). Esto muestra que dicha versión tiene una tendencia clara a subestimar el índice observado, puesto que los datos observados se reparten, principalmente, en las tres primeras categorías, como podemos ver en la tabla 5 y en la figura 5.a. Los errores más grandes aparecen al predecir la categoría Muy Buena cuando se observan las categorías Buena y Moderada (29,86 \% y 6,59\%).

Este comportamiento de la versión operativa del modelo en relación a la subestimación del material particulado es común en la mayoría de los modelos de transporte químico (Konovalov et al., 2009; STERN et al., 2008).

\begin{tabular}{|c|c|c|c|c|c|c|c|}
\hline & \multicolumn{5}{|c|}{ Observaciones } & \multirow[b]{2}{*}{ Total } \\
\hline & & Muy Buena & Buena & Moderada & Mala & Muy Mala & \\
\hline \multirow{5}{*}{ 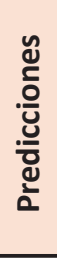 } & Muy Buena & 53,45 & 29,86 & 6,59 & 2,46 & 0,23 & 92,59 \\
\hline & Buena & 1,72 & 2,42 & 1,16 & 0,57 & 0,02 & 5,89 \\
\hline & Moderada & 0,25 & 0,44 & 0,24 & 0,14 & 0 & 1,07 \\
\hline & Mala & 0,1 & 0,22 & 0,06 & 0,07 & 0 & 0,45 \\
\hline & Muy Mala & 0 & 0 & 0 & 0 & 0 & 0 \\
\hline & Total & 55,52 & 32,94 & 8,05 & 3,24 & 0,25 & 100 \\
\hline
\end{tabular}

Tabla 5. Tabla de contingencia en porcentajes para el PM10 correspondiente a la versión operativa del modelo.

a)

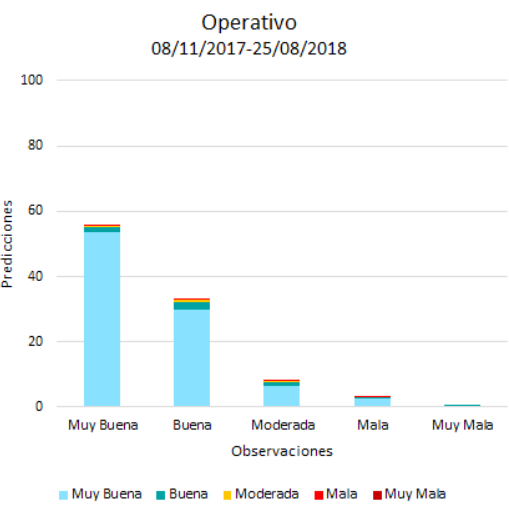

b)

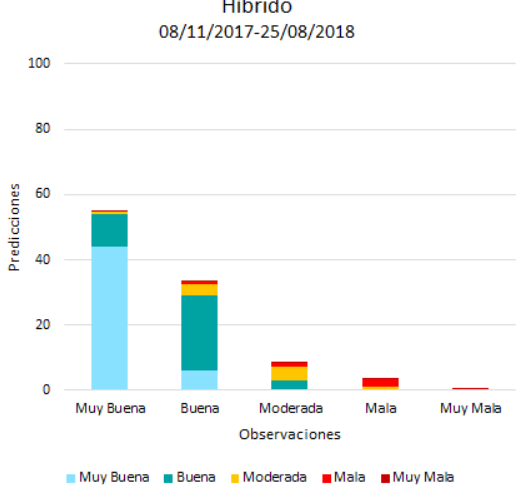

Figura 5.

Gráfico de barras

correspondiente a los datos de la verificación del PM10:

a) versión operativa del modelo y b) versión híbrida del modelo.

Por otro lado, con respecto a la versión híbrida del modelo (tabla 6 y figura 5.b), podemos decir que se aprecia una clara mejora en las predicciones con respecto a la versión operativa. El aumento de las predicciones 


\begin{tabular}{|c|c|c|c|c|c|c|c|}
\hline & \multicolumn{5}{|c|}{ Observaciones } & \multirow[b]{2}{*}{ Total } \\
\hline & & Muy Buena & Buena & Moderada & Mala & Muy Mala & \\
\hline \multirow{5}{*}{ 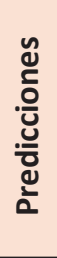 } & Muy Buena & 44 & 5,91 & 0,21 & 0,05 & 0,02 & 50,19 \\
\hline & Buena & 9,83 & 23,05 & 2,6 & 0,26 & 0,03 & 35,77 \\
\hline & Moderada & 0,87 & 3,61 & 4,28 & 0,89 & 0,01 & 9,66 \\
\hline & Mala & 0,01 & 0,68 & 1,1 & 2 & 0,07 & 4,08 \\
\hline & Muy Mala & 0,01 & 0,04 & 0,02 & 0,07 & 0,15 & 0,3 \\
\hline & Total & 54,95 & 33,29 & 8,21 & 3,27 & 0,28 & 100 \\
\hline
\end{tabular}

Tabla 6. Tabla de contingencia en porcentajes para el PM10 correspondiente a la versión híbrida del modelo.

correctas en las categorías Buena, Moderada y Mala hace que la proporción de predicciones correctas pase de ser 0,56 con la versión operativa a ser 0,73 con la versión híbrida (figura 8) y, además, que aumenten notablemente las probabilidades de detección en esas categorías, como podemos observar en la figura 9.

Sin embargo, como en el caso del $\mathrm{NO}_{2}$, las predicciones correctas disminuyen en la primera categoría y, como consecuencia, su probabilidad de detección es ligeramente menor con la versión híbrida del modelo que con la versión operativa (figura 9). En este caso, dado que la versión operativa está muy por debajo de la observación, las predicciones de la versión híbrida, en general, son mayores que las de la versión operativa. Por tanto, en las categorías Buena, Moderada y Mala la técnica empleada corrige correctamente la predicción original, pero en la categoría Muy Buena hay algunos casos que al aumentar demasiado la predicción híbrida, esta pasa a estar una categoría por encima de la observada cuando la predicción original era correcta.

\subsection{Resultados de la verificación para el PM2.5}

En el caso del PM2.5 las tablas de contingencia (tablas 7 y 8) muestran que tanto la versión operativa del modelo como la versión híbrida tienen un comportamiento parecido al que hemos visto anteriormente con el PM10. El porcentaje total de predicciones con la versión operativa (tabla 7 y figura 6.a) está repartido principalmente entre las categorías Mиу Buеnа у Buеna, pero en este caso la mayoría de las observaciones también están repartidas en esas dos categorías, lo que hace que los resultados sean ligeramente mejores que los que obtenemos con el PM10 para dicha versión.

Con respecto a la versión híbrida (tabla 8 y figura 6.b) vemos que, al igual que ocurre con el PM10, mejoran mucho los porcentajes de predicciones correctas en las categorías Buena, Moderada y Mala, por lo que la proporción de predicciones correctas es mayor que con la versión operativa y las probabilidades de detección de esas categorías mejoran mucho (figuras 8 y 9). La disminución del porcentaje de predicciones correctas en la categoría Muy Buena se debe al mismo motivo explicado anteriormente en el caso del PM10.

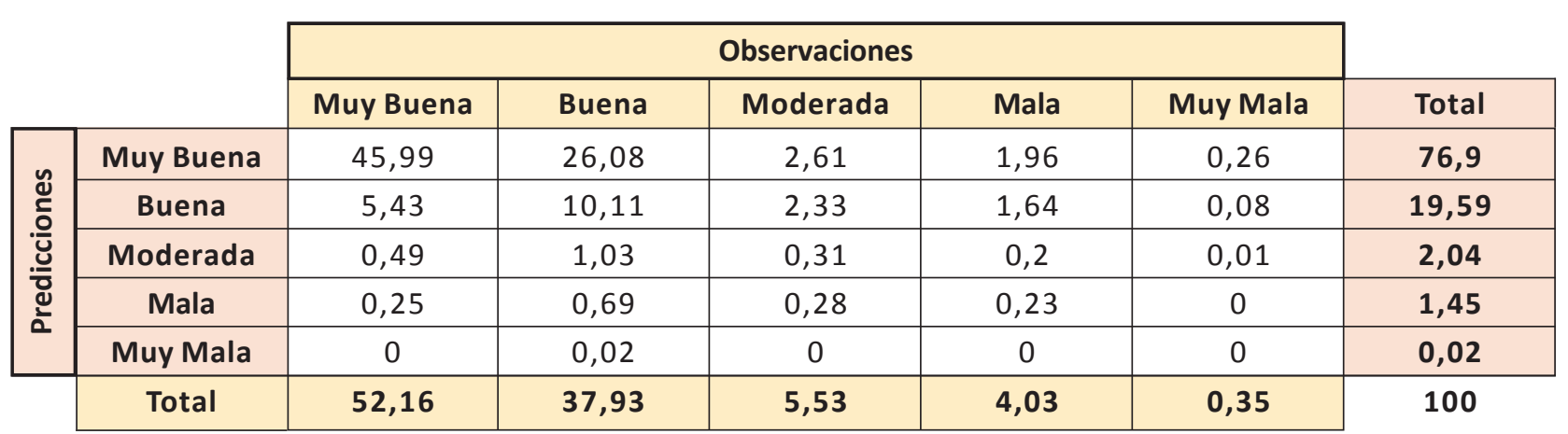

Tabla 7. Tabla de contingencia en porcentajes para el PM2.5 correspondiente a la versión operativa del modelo. 
a)

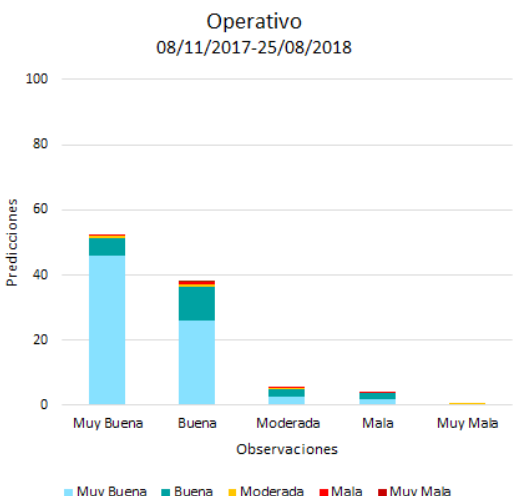

b)

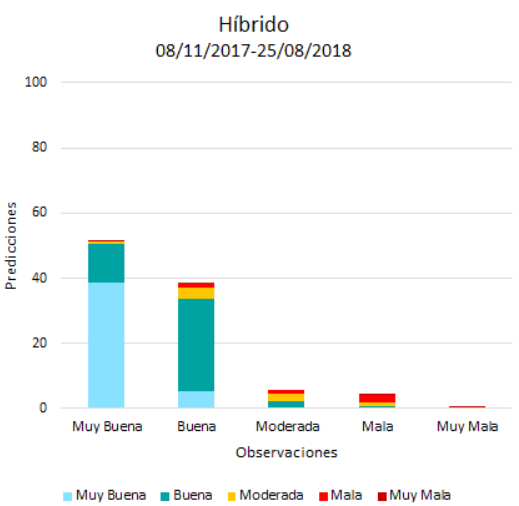

Figura 6.

Gráfico de barras correspondiente a los datos de la verificación del PM2.5: a) versión operativa del modelo y b) versión híbrida del modelo.

\begin{tabular}{|c|c|c|c|c|c|c|c|}
\hline & \multicolumn{5}{|c|}{ Observaciones } & \multirow[b]{2}{*}{ Total } \\
\hline & & Muy Buena & Buena & Moderada & Mala & Muy Mala & \\
\hline \multirow{5}{*}{ 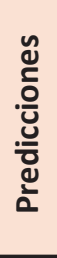 } & Muy Buena & 38,56 & 5,44 & 0,06 & 0,03 & 0,02 & 44,11 \\
\hline & Buena & 12,1 & 28,36 & 2,05 & 0,65 & 0,05 & 43,21 \\
\hline & Moderada & 0,53 & 3,18 & 2,35 & 1 & 0,1 & 7,16 \\
\hline & Mala & 0,3 & 1,27 & 1,2 & 2,34 & 0,1 & 5,21 \\
\hline & Muy Mala & 0,04 & 0,01 & 0 & 0,01 & 0,16 & 0,31 \\
\hline & Total & 51,53 & 38,26 & 5,66 & 4,12 & 0,43 & 100 \\
\hline
\end{tabular}

Tabla 8. Tabla de contingencia en porcentajes para el PM2.5 correspondiente a la versión híbrida del modelo.

\subsection{Resultados de la verificación para el $\mathrm{O}_{3}$}

Los resultados del ozono correspondientes a las tablas 9 y 10 muestran que el índice observado a lo largo de todo el año se encuentra principalmente entre las categorías Muy Buena, Buena y Moderada, aunque en esta última en menor medida (aproximadamente el 4,5\% de las observaciones) ya que los niveles de ozono observado más altos, en su mayoría, se registran solo en verano.

En la versión operativa del modelo (tabla 9 y figura 7.a), vemos que las predicciones de esta versión también están principalmente distribuidas en las categorías Muy Buena, Buena y Moderada, aunque tienen una clara tendencia a la sobrestimación. Así, los errores más destacados que se cometen son al predecir la categoría Buena cuando se observa Muy Buena $(24,13 \%)$ y al predecir Moderada cuando se observan las categorías Muy Buena y Buena $(2,79 \%$ y 9,11\%, respectivamente).

Por otro lado, atendiendo a los resultados de la versión híbrida del modelo (tabla 10 y figura 7.b), observamos que los porcentajes de aciertos en cada categoría del índice son claramente mayores que los de la versión operativa. Así por ejemplo, el porcentaje de predicciones correctas en la categoría Muy Buena pasa de ser un $24,71 \%$ con la versión operativa a un 35,67 \% con la versión híbrida. Como consecuencia, los errores que se cometen con la versión operativa son más pequeños que con la versión híbrida. De estos podemos destacar el error al predecir la categoría Buena cuando se observa Muy Buena que, como hemos mencionado anteriormente, supone el $24,13 \%$ de los datos con la versión operativa y pasa a ser un 14,61\% con la versión híbrida.

La disminución de los errores y el aumento de los aciertos al utilizar la versión híbrida del modelo se ve reflejada en el aumento de la proporción de predicciones correctas que pasa de ser 0,53 a 0,68 (figura 8). 
Igualmente las probabilidades de predicción de cada categoría mejoran notablemente (figura 9). Cabe destacar que, a diferencia de como ocurre con el material particulado y el $\mathrm{NO}_{2}$, en el caso del ozono no hay ninguna categoría del índice en la que la versión híbrida tenga porcentajes menores de aciertos que la versión operativa.

El ozono es un contaminante secundario, ya que se forma a partir de ciertos precursores como son los óxidos de nitrógeno $\left(\mathrm{NO}_{\mathrm{x}}\right)$, el monóxido de carbono $(\mathrm{CO})$ o los compuestos orgánicos volátiles $(\mathrm{COV})$, entre otros. Esta variedad de fuentes y especies precursoras junto con una dinámica compleja (altamente no lineal) hacen que su modelización sea complicada y, por tanto, que los niveles previstos del índice de ozono por el modelo operativo (sobre todo en verano) no se correspondan en muchos casos con los valores observados del índice.

\begin{tabular}{|c|c|c|c|c|c|c|c|}
\hline & & \multicolumn{5}{|c|}{ Observaciones } & \multirow[b]{2}{*}{ Total } \\
\hline & & Muy Buena & Buena & Moderada & Mala & Muy Mala & \\
\hline \multirow{5}{*}{ 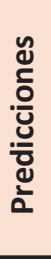 } & Muy Buena & 24,71 & 7,59 & 0,31 & 0 & 0 & 32,61 \\
\hline & Buena & 24,13 & 26,81 & 2,09 & 0 & 0,01 & 53,04 \\
\hline & Moderada & 2,79 & 9,11 & 1,88 & 0 & 0 & 13,78 \\
\hline & Mala & 0,05 & 0,27 & 0,16 & 0 & 0 & 0,48 \\
\hline & Muy Mala & 0,01 & 0,05 & 0,03 & 0 & 0 & 0,09 \\
\hline & Total & 51,69 & 43,83 & 4,47 & 0 & 0,01 & 100 \\
\hline
\end{tabular}

Tabla 9. Tabla de contingencia en porcentajes para el $\mathrm{O}_{3}$ correspondiente a la versión operativa del modelo.

\begin{tabular}{|c|c|c|c|c|c|c|c|}
\hline & & \multicolumn{5}{|c|}{ Observaciones } & \multirow[b]{2}{*}{ Total } \\
\hline & & Muy Buena & Buena & Moderada & Mala & Muy Mala & \\
\hline \multirow{5}{*}{ 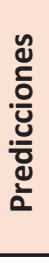 } & Muy Buena & 35,67 & 6,32 & 0,08 & 0 & 0 & 42,07 \\
\hline & Buena & 14,61 & 29,39 & 1,36 & 0 & 0 & 45,36 \\
\hline & Moderada & 0,79 & 8,35 & 2,99 & 0 & 0 & 12,13 \\
\hline & Mala & 0 & 0,17 & 0,23 & 0 & 0 & 0,4 \\
\hline & Muy Mala & 0,04 & 0,01 & 0,01 & 0 & 0,01 & 0,04 \\
\hline & Total & 51,08 & 44,24 & 4,67 & 0 & 0,01 & 100 \\
\hline
\end{tabular}

Tabla 10. Tabla de contingencia en porcentajes para el $\mathrm{O}_{3}$ correspondiente a la versión híbrida del modelo.

a)

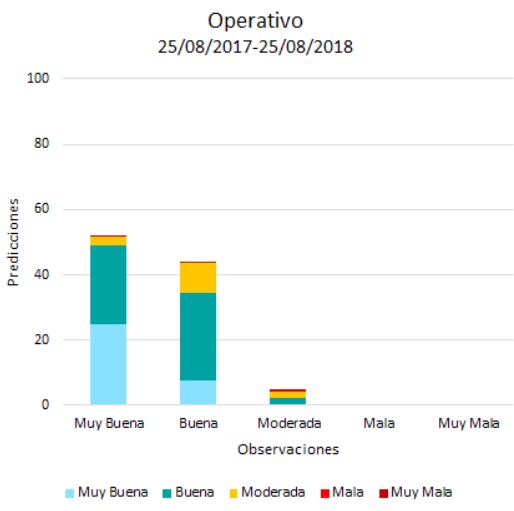

b)

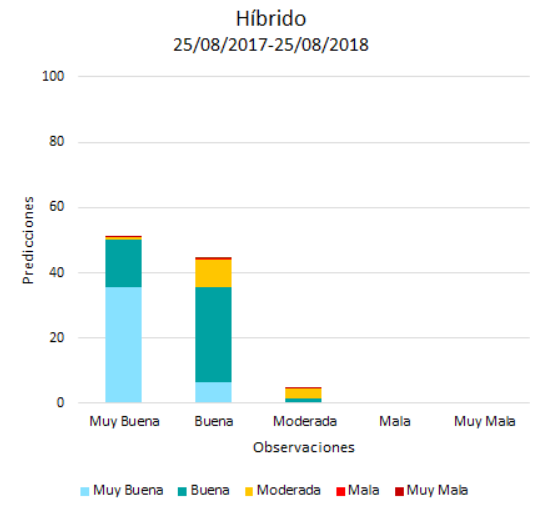

Figura 7.

Gráfico de barras correspondiente a los datos de la verificación del $\mathrm{O}_{3}$ : a) versión operativa del modelo y b) versión híbrida del modelo. 
a)

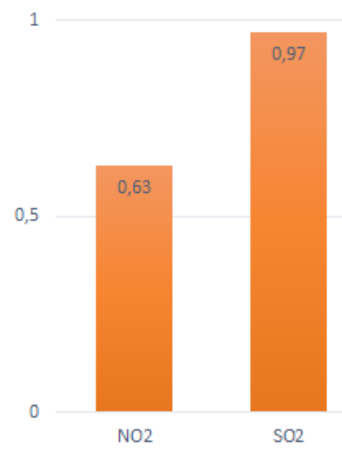

Operativo

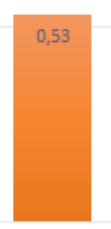

O3 b)

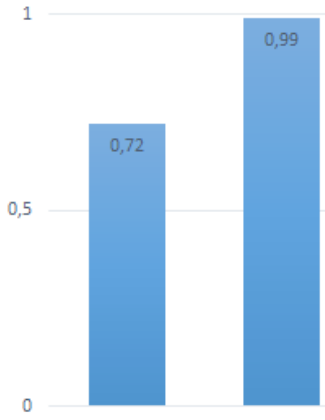

$\mathrm{SO} 2$
Híbrido

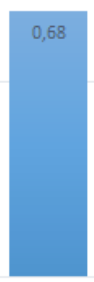

03

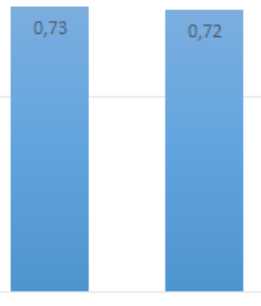

PM10

PM2.5

Figura 8. Proporción de predicciones correctas para cada contaminante considerado en el índice de calidad del aire: a) versión operativa del modelo y b) versión híbrida del modelo.
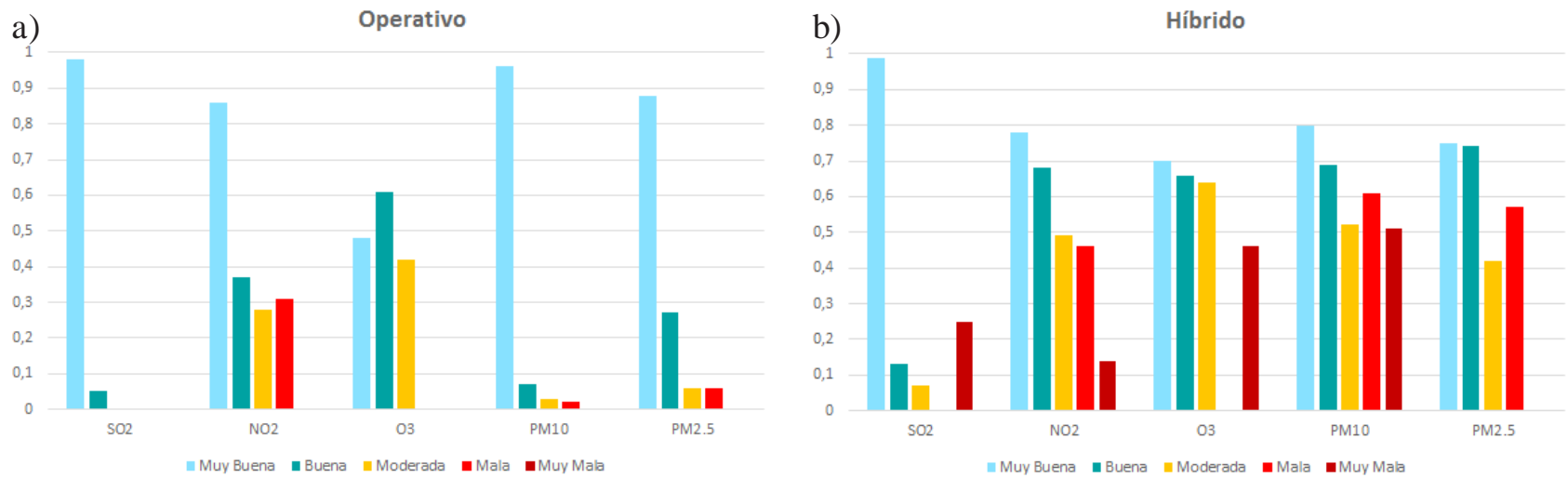

Figura 9. Probabilidad de predicción de cada categoría del índice, para cada contaminante: a) versión operativa del modelo y b) versión híbrida del modelo.

\section{CONCLUSIONES}

Los resultados de la verificación del índice de calidad del aire previsto por la versión operativa del modelo y por la versión híbrida nos indican que la implementación de un postproceso basado en la técnica Hybrid Forecast supone una mejora considerable en la calidad de las predicciones en cada especie considerada en el índice $\left(\mathrm{NO}_{2}, \mathrm{SO}_{2}, \mathrm{PM} 10, \mathrm{PM} 2.5\right.$ y $\left.\mathrm{O}_{3}\right)$. Los principales contaminantes en los que podemos decir que la mejora es más notable son el ozono $\left(\mathrm{O}_{3}\right)$ y el material particulado de tamaño menor de 10 micras (PM10), que precisamente son dos de los contaminantes que presentan un mayor sesgo y una gran homogeneidad espacial.

Sin embargo, es necesario mejorar esta metodología para poder suavizar los errores de las observaciones que hacen que la predicción de algunas categorías del índice empeore, como por ejemplo ocurre con el $\mathrm{NO}_{2}$, el PM10 y el PM2.5 en la categoría Muy Buena. Considerar el residuo de un conjunto de estaciones con características similares o un promedio temporal de los residuos pueden ser dos opciones a emplear para evitar dichos errores.

\section{AGRADECIMIENTOS}

Por último, queremos agradecer al Ministerio de Agricultura y Pesca, Alimentación y Medio Ambiente habernos permitido acceder a los datos de las estaciones de observación, proporcionados por las diferentes redes nacionales, autonómicas y locales de calidad del aire, para la realización de este trabajo. 


\section{REFERENCIAS}

JolifFe, I. T. y StePHENSON, D. B., 2012. Forecast Verification: A practicioner's Guide in Atmospheric Science 2nd ed. Wiley-Blackwell, 274 pp.

Josse, B., Simon, P. y Peuch, V.-H., 2004. Radon global simulations with the multiscale chemistry and transport model MOCAGE. Tellus, 56B, 339-356.

Kang, D., Mathur, R., RaO, S. T. y ShaOcai, Y., 2008. Bias adjustment techniques for improving ozone air quality forecasts. J. Geophys. Res., 113 (D23308).

Konovalov, I. B., Beekmann, M., Meleux, F., Dutot, A. y Foret, G., 2009. Combining deterministic and statistical approaches for PM10 forecasting in Europe. Atmospheric Environment, 43, 6425-6434.

Stern, R., Builtjes, P., Schaap, M., Timmermans, R., Vautard, R., Hodzic, A., Memmesheimer, M., Feldmann, H., Renner, E., Wolke, R. y Kerschbaumer, A., 2008. A model inter-comparison study focusing on episodes with elevated PM10 concentrations. Atmospheric Environment, 42, 4567-4588.

Stockwell, W. R., KiRChNer, F., Kuhn, M. y Seefeld, S., 1997. A new mechanism for regional atmospheric chemistry modelling. Journal of Geophysical Research, 102, 25847-25879. 
